\title{
Analyses électrochimiques des eaux de pluie provoquées : cas du Burkina Faso
}

\author{
Maazou Ahmed MAMAN ${ }^{1 *}$, Jacques SAWADOGO ${ }^{3}$, Dasmané BAMBARA $^{3}$, \\ Moussa BOUGOUMA ${ }^{4}$ et Jean Boukari LEGMA ${ }^{2}$ \\ ${ }^{1}$ Université de Tillabéri, Institut Universitaire de Technologie, B.P. 10662 Niamey, Niger. \\ ${ }^{2}$ Université Saint- Thomas- d'Aquin (USTA), Faculté des Sciences et Technologies, \\ 06 BP 10212 Ouagadougou 06, Burkina Faso. \\ ${ }^{3}$ Centre National de la Recherche Scientifique et Technologique/Institut de l'Environnement et de Recherches \\ Agricoles (CNRST/INERA), Laboratoire Sol-Eau-Plante, 01 BP 476 Ouagadougou 01, Burkina Faso. \\ ${ }^{4}$ Université de Koudougou, Faculté de Sciences et Techniques, BP 376 Koudougou, Burkina Faso. \\ *Auteur correspondant, E-mail : maazou2000@gmail.com, Tel : +22799542013
}

\section{REMERCIEMENTS}

Ce travail a été financé par "Programme SAAGA» de la base aérienne du Burkina Faso. Les auteurs expriment leurs remerciements pour l'appui technique, matériel et financier dont ce travail a bénéficié.

\section{RÉSUME}

La problématique de l'accès à l'eau constitue une préoccupation majeure pour tous, du fait qu'elle est étroitement liée à la santé et au développement socio-économique. L'accès à l'eau pour les populations rurales des pays en développement reste un défi à relever. Au Burkina Faso, malgré les efforts fournis par l'Etat et ses partenaires au développement, la rareté de la ressource demeure une réalité. Dans le cadre d'une gestion participative des eaux de pluie provoquées au Burkina Faso, un suivi de la qualité des eaux a été réalisé en campagne pluvieuse. Cette étude fait l'état des lieux de l'impact des opérations d'ensemencement des nuages du PROGRAMME SAAGA sur la pollution de l'environnement au Burkina Faso. L'objectif du travail est de déterminer le niveau de concentration en ions calcium $\left(\mathrm{Ca}^{2+}\right)$, iodures $\left(\mathrm{I}^{-}\right)$et argent $\left(\mathrm{Ag}^{+}\right)$dans les eaux de pluie issues des opérations d'ensemencement des nuages. Les résultats des analyses faites par Ionométrie et par voltamétrie cyclique montrent que les concentrations moyennes mesurées en ions calcium varient entre 1,415 $\mathrm{mg} / \mathrm{l}$ et $6,550 \mathrm{mg} / \mathrm{l}$, celles d'iodures ( $\left.\mathrm{I}^{-}\right)$est pratiquement négligeables de même que les ions $\operatorname{argent}\left(\mathrm{Ag}^{+}\right)$. D'une manière générale, les résultats obtenus par analyse électrochimique des eaux de pluie provoquées prélevées sur les différents sites d'études montrent que les opérations d'ensemencement des nuages n'ont aucun impact sur les eaux et sur l'environnement.

(C) 2017 International Formulae Group. All rights reserved.

Mots clés : Eaux de pluie, Ensemencement des nuages, Programme SAAGA, Ionométrie, voltamétrie cyclique, Burkina Faso. 


\title{
Electrochemical analysis of rainwater caused: cas of Burkina Faso
}

\begin{abstract}
The problematic of the access to water constitutes a major concern for all, because of its strong linkage to health and to socio-economic development. The Access to water for rural populations in developing countries remains a challenge for the sustainable development despite the efforts deployed by the government and its partners to achieve the Millennium development goals, the lack of water remains a reality especially in Burkina Faso. As part of a participatory management of rainwater caused in Burkina Faso, monitoring the water quality was carried in the rainy season. This study is an inventory of the impact of clouds seeding program of "PROGRAMME SAAGA" on environmental pollution in Burkina Faso. The objective of this study is to determine the level of concentration of the calcium ions $\left(\mathrm{Ca}^{2+}\right)$, iodide (I-) and silver $\left(\mathrm{Ag}^{+}\right)$in rainwater resulting from cloud-seeding operations. The results of the analysis made by ionometry and cyclic voltammetry indicated that calcium concentrations range from $1.415 \mathrm{mg} / 1$ to $6.550 \mathrm{mg} / \mathrm{l}$, those of iodides $\left(\mathrm{I}^{-}\right)$and silver ${ }^{\prime} \mathrm{Ag}^{+}$) are almost negligible. In general, the results obtained by electrochemical analysis of the rainwater collected from different sites of studies show that cloud-seeding operations do not have any armful impact on water and the environment.
\end{abstract}

(C) 2017 International Formulae Group. All rights reserved.

Keywords: Rainwater, Cloud -seeding, PROGRAMME SAAGA, Ionometry, cyclic voltammetry, Burkina Faso.

\section{INTRODUCTION}

L'eau constitue l'une des ressources naturelles les plus importantes pour la survie de l'humanité et le développement socioéconomique des nations. Cet élément indispensable à la vie des êtres vivants peut être aussi une source de maladies, car il véhicule d'innombrables substances chimiques telles que les ions calcium, argent et iodure. Selon certains auteurs (Organisation Mondiale de la Santé/UNICEF, 2007; Journal Officiel de la République Française, 2008; De Gouvello, 2011; Beniston, 2011), 1,1 milliard de personnes n'ont pas accès à une eau de saine dont $84 \%$ vivant dans les zones rurales. Aussi l'Afrique subsaharienne reste la région où la situation demeure la plus préoccupante, car entre 1990 et 2004, le nombre de personnes n'ayant pas accès à l'eau y a augmenté de $23 \%$. Selon d'autres estimations (Kissinger et al., 1984; Organisation Mondiale de la Santé/, 2000; Dianou et al., 2003; Dianou et al., 2011; Nitiema et al., 2013), cette situation entraine chaque année la mort de 1,6 million d'enfants et près de 4 millions d'êtres humains par des maladies liées à l'eau et à l'environnement. Comme le souligne aussi Klaus Toepfler, directeur général du Programme des Nations Unies pour l'Environnement (PNUE), cité par Kouam et al., 2006, "L'eau est étroitement liée à la santé, l'agriculture (sol), l'énergie et la biodiversité. Sans progrès dans le domaine de l'eau, il sera difficile, voire impossible, d'atteindre les autres objectifs du millénaire pour le développement. En effet, maints pays africains situés dans des zones arides ou semiarides tel que le Burkina Faso sont confrontés au phénomène de sécheresse, entraînant ainsi des problèmes d'approvisionnement en eau. Pour y faire face, certains d'entre eux ont recours à l'ensemencement des nuages provoquant ainsi les précipitations en modifiant le temps. En Afrique subsaharienne, le Burkina Faso fait partie des pays qui se sont très tôt intéressés à cette technique d'ensemencement des nuages qui est une technique de précipitation des nuages. C'est ainsi qu'en 1998, une opération dénommée "PROGRAMME SAAGA" fut déclenchée suite à un important déficit pluviométrique enregistré en 1997. Cette opération a bénéficié de l'appui technique et logistique du Royaume du Maroc qui pratique cette activité depuis les 
années $1980 . \quad$ Cette technique d'ensemencement consiste à introduire dans les nuages des sels de calcium et d'iodure d'argent afin d'accélérer le processus de précipitation. La présente étude se situe dans ce cadre et a pour but d'évaluer la qualité chimique des eaux de pluie provoquées par l'ensemencement des nuages sur une campagne pluvieuse. A cet effet, le niveau de concentration des ions calcium $\left(\mathrm{Ca}^{2+}\right)$, iodure $\left(\mathrm{I}^{-}\right)$, et argent $\left(\mathrm{Ag}^{+}\right)$a été déterminé dans des échantillons d'eaux de pluie collectés; par l'utilisation des méthodes fines d'analyses électrochimiques. Pour mener à bien ce travail, les méthodes d'analyses par potentiométrie à intensité nulle et la voltamétrie cyclique associée à la technique des ajouts dosés ont été utilisées.

\section{MATERIEL ET METHODES}

Présentation des sites d'étude

Situé entre le désert du Sahara et la forêt équatoriale africaine plus précisément dans la boucle du Niger, au Nord du golfe de Guinée, le Burkina Faso est un pays enclavé d'une superficie de $274200 \mathrm{~km}^{2}$. Le Burkina Faso possède un climat tropical de type soudano-sahélien avec deux saisons très contrastées : la saison des pluies et des saisons sèches. Le pays est régulièrement soumis à de nombreuses sécheresses et c'est ainsi que fut créé l'opération SAAGA (pluie en langue moré). Le réseau de mesure de la qualité des eaux de pluie provoquées par l'opération SAAGA est constitué de treize (13) sites répartis suivant six (6) axes du Burkina Faso (Figure 1). Le choix de ces sites a tenu compte de la couverture de l'ensemble du territoire national du pays.

\section{Prélèvement et conservation des Échantillonnages}

Le prélèvement d'un échantillon d'eau de pluie est une opération assez délicate à laquelle le plus grand soin doit être apporté. Il conditionne les résultats analytiques et l'interprétation qui en sera donnée. L'échantillon doit être homogène, représentatif et obtenu sans modifier les caractéristiques physico-chimiques de l'eau (Rodier et al., 2009). Il est donc nécessaire de développer une méthodologie adaptée à chaque cas et utiliser le matériel convenable. Ces échantillons d'eau ont été collectés avec une fréquence de 30 jours sur une période de quatre (4) mois (juin à septembre), représentant la saison pluvieuse au Burkina Faso.

Le matériel de prélèvement a fait l'objet d'une attention particulière. Ainsi, des flacons neufs en polyéthylène ont été employés. Avant la sortie sur le terrain, les flacons sont d'abord lavés à grande eau, puis avec de l'acide nitrique $10 \%$ et finalement rincés abondamment avec de l'eau distillée (Chippaux et al., 2002; Rosillon et al., 2012; Sunda Makuba, 2012; Kabore et al., 2013). Sur le terrain avant remplissage des flacons, par mesure de sécurité, elles sont lavées trois (03) fois avec l'eau à analyser, puis remplis jusqu'au bord. Les échantillons prélevés, soigneusement étiquetés et conservés à $5{ }^{\circ} \mathrm{C}$ dans des glacières sont ensuite transportés au laboratoire pour les autres analyses électrochimiques. Ces échantillons ont été protégés contre les rayonnements solaires durant le transport (Altaher et al., 2011; Rosillon et al., 2012; Savadogo et al., 2013) pour éviter toute décomposition anaérobie.

\section{Analyse des eaux de pluie provoquées Dosage par ionométrie}

L'ionométrie consiste à déterminer par la potentiométrie à intensité nulle, les concentrations des espèces ioniques en solution. On mesure une différence de potentiel entre une électrode indicatrice et une électrode de référence à potentiel fixe. Les électrodes indicatrices utilisées sont sélectives et spécifiques à un ion donné (Shain et al., 1961; Fourcade et al., 2000; Maman, 2008; Kabore et al., 2013a; Kabore et al., 2013b). Cette méthode a été utilisée pour la détermination des teneurs des ions iodures et calcium dans les échantillons d'eau de pluie provoquée. 


\section{Mode opératoire}

$\mathrm{Au}$ laboratoire de Chimie des Matériaux et Moléculaires de l'Université de Ouaga I Professeur Joseph KI-ZERBO, $20 \mathrm{ml}$ de l'échantillon d'eau ont été prélevés et introduit dans un bécher de $100 \mathrm{ml}$, puis $20 \mathrm{ml}$ d'un électrolyte support (acide perchlorique) ont été additionnés. Enfin, le potentiel E pour chaque échantillon a été mesuré (Mathieu et al., 1971; Ahoussi et al., 2010).

\section{Appareillage}

Deux paramètres physico-chimiques (le $\mathrm{pH}$ et la température) ont été mesurés in situ, immédiatement après la tombée de la pluie (prélèvement de l'échantillon) dans chaque zone de l'étude. Le pH est mesuré sur le terrain à l'aide d'un $\mathrm{pH}$-mètre portatif de marque Radiometer Analytical pHM201 et au laboratoire avec un pH-mètre de laboratoire de marque Hanna pH209. La prise de la température s'est effectuée à l'aide d'un thermomètre à affichage numérique. Les mesures potentiométriques ont été réalisées à l'aide d'un pH-mètre millivoltmètre numérique, d'un agitateur magnétique type Rotamag 10 et de différentes électrodes sélectives.

\section{Les électrodes}

La détermination des ions spécifiques contenus dans les échantillons d'eaux de pluie a été réalisée à l'aide de différentes électrodes spécifiques suivantes : une électrode sélective des ions iodures ISE 25I; une électrode sélective des ions calcium ISE $25 \mathrm{Ca}$ et une électrode de référence REF 251. Toutes les électrodes utilisées sont de marque Radiometer Analytical.

\section{Dosage par voltamétrie cyclique couplé à la méthode des ajouts dosés La voltamétrie cyclique}

La voltampérométrie cyclique est une méthode d'analyse largement utilisée dans l'étude des processus électrochimiques. Elle permet d'acquérir rapidement différents types d'informations, notamment sur la thermodynamique des réactions d'oxydoréduction, sur la cinétique de transfert d'électron, sur les phénomènes impliquant l'adsorption.
Cette méthode consiste en un balayage linéaire du potentiel entre une borne initiale et une borne finale. Ce potentiel est mesuré entre l'électrode de travail et l'électrode de référence. Les courbes intensité-potentiel $(\mathrm{i}=$ $\mathrm{f}(\mathrm{E})$ ) obtenues sont appelées voltamogrammes.

\section{La méthode des ajouts dosés}

Cette technique consiste à ajouter à l'espèce à analyser une petite quantité d'une solution de concentration connue en ion étudié, ce qui entraîne une variation $\Delta \mathrm{C}$ de la concentration de l'ion et par conséquent celle de la hauteur du pic. La concentration $\mathrm{C}$ de l'espèce à analyser est déterminée par l'équation 1 ci-dessous (Rosillon et al., 2012; Kabore et al., 2013; Sawadogo et al., 2015)

$$
\begin{aligned}
& C=\frac{\mathrm{i}_{1}}{\mathrm{i}_{2}-\mathrm{i}_{1}} \Delta \mathrm{C} \\
& \mathrm{i}_{1} \text { intensité du pic avant l' ajout } \\
& \mathrm{i}_{2} \text { intensité du pic après ajout }
\end{aligned}
$$

\section{Mode opératoire}

Pour les mesures de la voltamétrie cyclique, $10 \mathrm{ml}$ d'échantillon d'eau ont été introduit dans une cellule de mesure électrochimique puis après un ajout de $10 \mathrm{~mL}$ d'un électrolyte support $\left(\mathrm{HClO}_{4} \quad 0,1 \mathrm{M}\right)$, un barbotage à l'azote pendant 15 minutes pour désoxygéner la solution a été effectué. Les enregistrements du voltamogramme ont été effectués sur un intervalle de $-300 \mathrm{mV}$ à 200 $\mathrm{mV}$ avec une vitesse de balayage de $3 \mathrm{mV} / \mathrm{s}$.

\section{La température de l'eau de pluie}

C'est très important de connaître la température de l'eau avec une bonne précision. En effet, celle-ci joue un rôle dans la solubilité des gaz, dans la dissociation des sels dissous et dans la détermination du $\mathrm{pH}$ et les mélanges éventuels (Vialle et al., 2011). D'une façon générale, la température des eaux est influencée par l'origine dont elles proviennent (Vialle et al., 2010; Breton et al., 2010; Sablayrolles et al., 2011).

\section{Le Potentiel d'hydrogène (pH)}

Le potentiel Hydrogène $(\mathrm{pH})$ mesure la concentration en ions $\mathrm{H}^{+}$de l'eau. Il traduit ainsi la balance entre acide et base sur une échelle de 0 à 14 et 7 étant le $\mathrm{pH}$ de 
neutralité. Ce paramètre conditionne un grand nombre d'équilibres physico-chimiques, et dépend de facteurs multiples, dont la température et l'origine de l'eau, il représente une indication importante en ce qui concerne l'agressivité de l'eau.

\section{Appareillage}

L'ensemble des mesures électrochimiques a été mené dans une cellule à trois électrodes connectée à un stand polarographique de type MDE 150 . Une électrode à disque tournant à embout d'argent de type EM-EDI-AG (diamètre $5 \mathrm{~mm}$ ) marque Tacussel et reliée à une unité de contrôle de vitesse CTV 101 a été utilisée comme électrode de travail (ET). Une électrode de référence $(\mathrm{ER})$ au chlorure d'argent type TR020 et une électrode auxiliaire (EA) de platine type TM020 toutes deux de marque Radiometer. Le stand polarographique est connecté à un potentiostat PST 050 de marque Radiometer analytical dont le rôle est de maintenir à une valeur déterminée (fixe ou variable) le potentiel d'une électrode. L'acquisition et le traitement des données se font grâce au logiciel électrochimique "voltamaster4", Le dispositif expérimental utilisé est représenté à la Figure 2.

\section{Analyses statistiques}

Les données obtenues ont été soumises à une analyse de variance à un facteur et les différences entre les moyennes observées entre sites et périodes ont été comparées suivant le test de Newman-Keuls au seuil de $5 \%$ avec le logiciel XLSTAT Pro 7.5.2. Les corrélations au seuil de $5 \%$ entre les différentes variables ont été analysées en relation avec les périodes d'échantillonnage et les sites. Les paramètres mesurés ont été évalués suivant les normes du Système d'Evaluation de la Qualité de l'Eau des cours d'eau (SEQ - Eau, France 2003). Les normes OMS (World Health Organization, 2004) pour la qualité des eaux de boisson ont également été prises en compte du fait que les eaux de pluie alimentent également certaines populations (Koukounari et al., 2007; Sanou et al., 2016) du Burkina Faso.

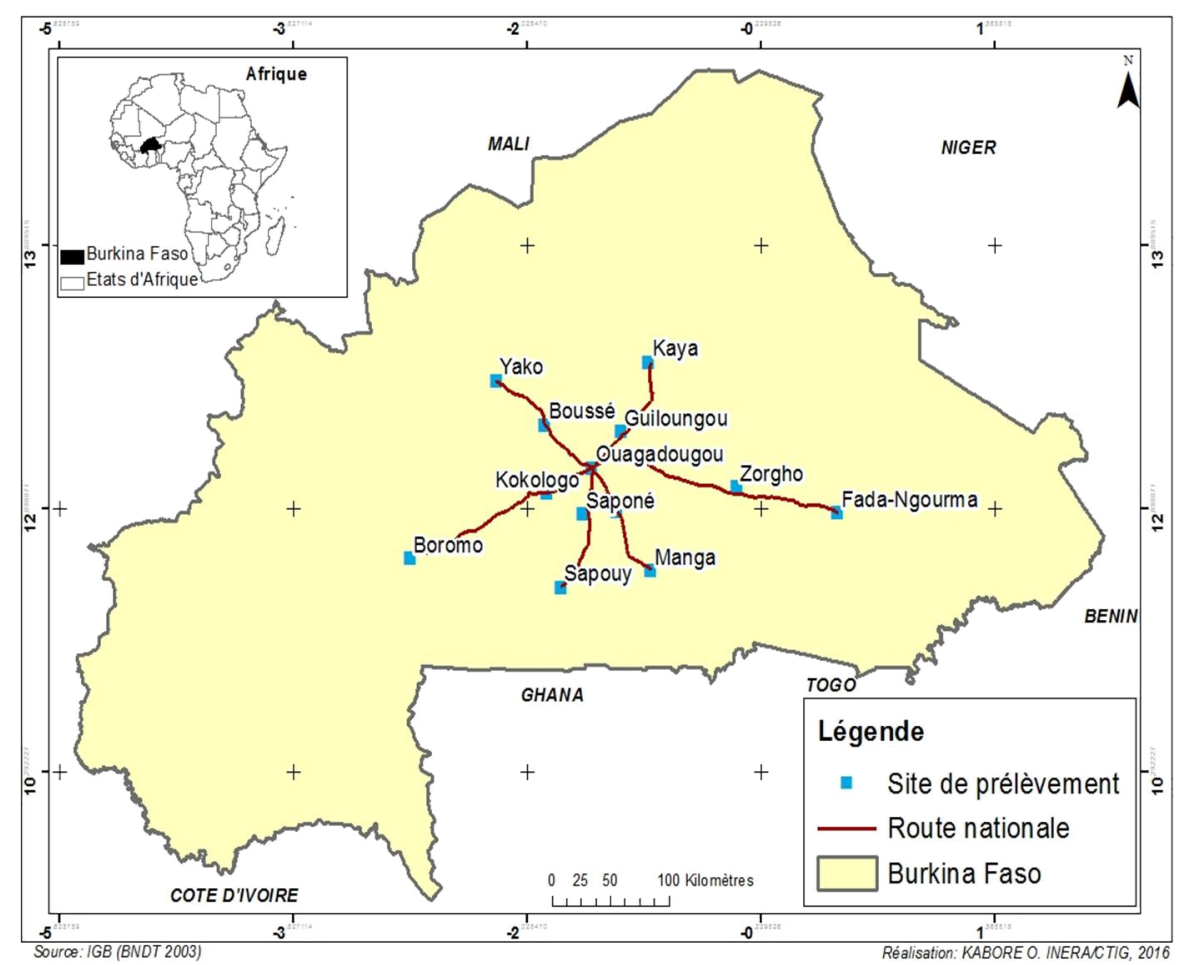

Figure 1 : Sites de prélèvements des échantillons d'eau de pluie provoquée. 


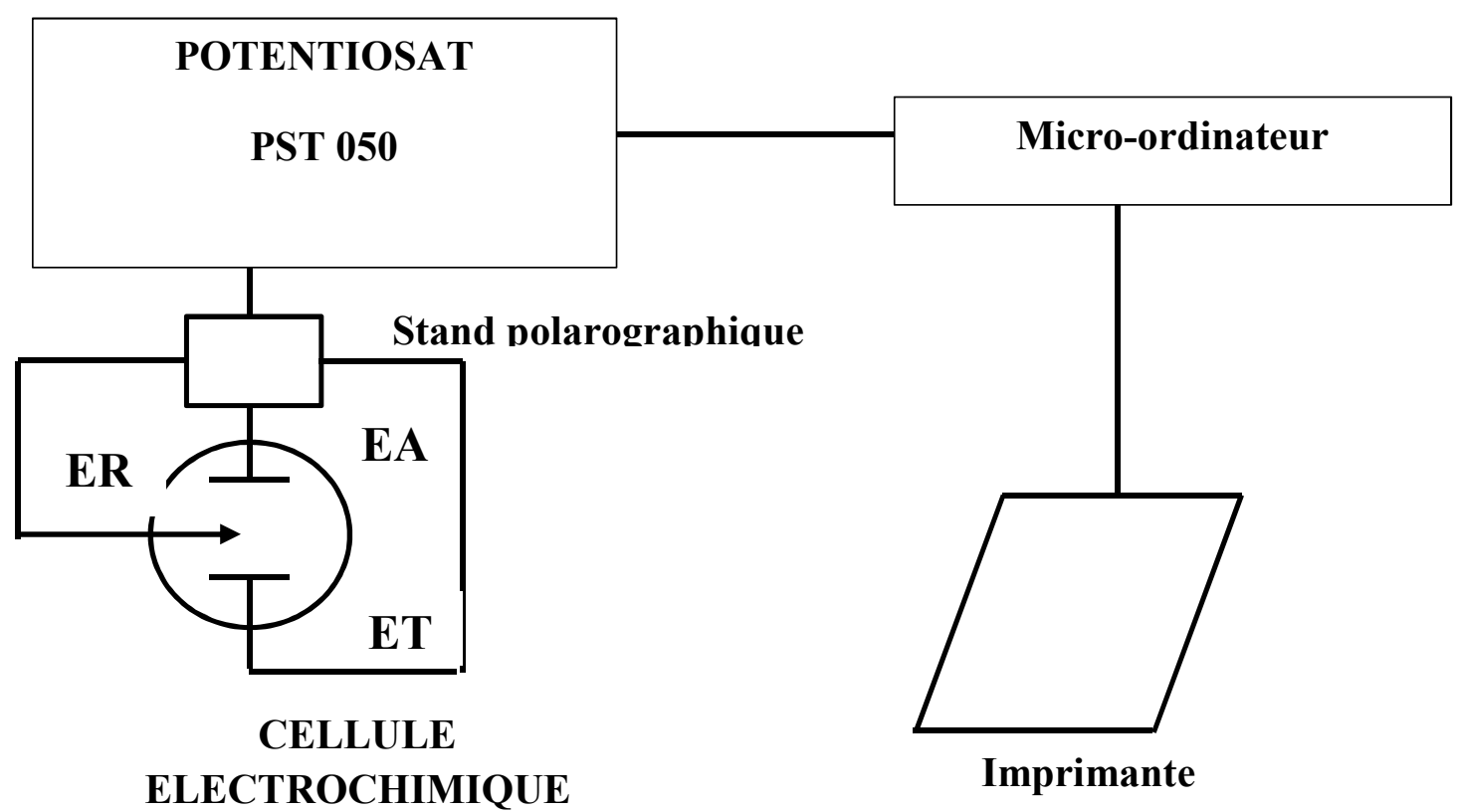

Figure 2 : Dispositif expérimental pour la mesure des paramètres électrochimiques.

\section{RÉSULTATS}

Les résultats obtenus sont représentés sur les Figures 3 et 4 pour les valeurs relevées de paramètres de température et de $\mathrm{pH}$. Les résultats des concentrations observées en ions calcium $\left(\mathrm{Ca}^{2+}\right)$ et paramètres chimiques sont consignés dans les Tableaux 2 et 3 .

\section{La température de l'eau de pluie}

Les mesures de températures (Figure 3) répétées ont été effectuées sur le terrain dès la fin de la pluie à intervalles réguliers. L'étude a révélé des valeurs de températures relativement basses durant la période de prélèvement et ces températures ne présentent pas de grandes variations d'un site à un autre. La valeur minimale moyenne est de $36{ }^{\circ} \mathrm{C}$ et la valeur maximale moyenne est de $38,2{ }^{\circ} \mathrm{C}$ pour les différents échantillonnages.

\section{Le Potentiel d'hydrogène (pH)}

Les mesures de $\mathrm{pH}$ (Figure 4) répétées ont été effectuées à l'aide d'un pH-mètre électronique portatif et une électrode combinée, étalonnée entre chaque mesure avec les tampons 4 et 7 en quelques secondes pour les eaux de pluie du Burkina Faso. La plupart des échantillons prélevés ont un $\mathrm{pH}>$ 7. En effet, les valeurs moyennes du $\mathrm{pH}$ se situent entre 6,5 et 8,5 pour les eaux potables (Lautenschlager et al., 2010; Kabore et al., 2013b; Houelome et al., 2016). Les échantillons d'eau (Figure 4) sont pratiquement basiques et se situent presque tous dans la plage de $\mathrm{pH}$ recommandé par l'OMS (6,5 - 8) (Abdoulkadri et al., 2015).

\section{Paramètres électrochimiques \\ Ions calcium $\left(\mathrm{Ca}^{2+}\right)$ par potentiométrie}

Le calcium est un métal alcalinoterreux extrêmement répandu dans la nature et en particulier dans les roches calcaires sous forme de carbonates. C'est le composant majeur de la dureté de l'eau. Les valeurs des potentiels (Tableau 1) mesurés dans les solutions étalons à différentes concentrations connues ont permis le tracé de la courbe d'étalonnage de la Figure 5.

Cette courbe d'étalonnage obtenue étant très proche de la linéarité avec un coefficient de corrélation $\mathrm{R}^{2}=0,998$, elle est donc fiable pour la détermination des 
concentrations en ions calcium des échantillons d'eau de pluie provoquée. L'exploitation de cette courbe donne les résultats (Tableau 2) qui sont une moyenne de cinq mesures. Les analyses en ions calcium des échantillons d'eau de pluie provoquée sont encore trop peu nombreuses au Burkina Faso, mais donnent des concentrations moyennes variant entre $1,415 \mathrm{mg} / \mathrm{l}$ et 6,550 $\mathrm{mg} / \mathrm{l}$ (Tableau 2). Aucune de ces eaux ne présente donc de concentration supérieure à la norme OMS qui est de $100 \mathrm{mg} / \mathrm{l}$ (Abdoulkadri, et al., 2015). Les Tableaux 2 et 3 présentent les analyses de variance et les paramètres physico-chimiques des échantillons d'eau de pluie pour les quatre mois de saison pluvieuse. Dans l'ensemble, les résultats indiquent une faible concentration en ions calcium dans tous les échantillons d'eau de pluies provoquées aux différents sites au regard des normes de qualité pour les eaux (Turton et al., 2000; Cheng et al., 2005; Koukounari et al., 2007; Yao et al., 2016).

\section{Ions iodure (I) par potentiométrie}

L'étalonnage de l'électrode spécifique aux ions iodures donne les potentiels consignés dans le Tableau 1 ci-dessous. La mesure des potentiels des solutions étalons permet de tracer la courbe d'étalonnage (Figure 6) linéaire avec un coefficient de corrélation $\mathrm{R}^{2}=1$, donc très fiable pour la détermination des concentrations des ions iodures. Les différentes mesures (Tableau 2) de potentiel effectuées sur les échantillons d'eau des différentes localités du pays ont donné des valeurs de potentiel comprises entre $38,86 \mathrm{mV}$ et $66,59 \mathrm{mV}$. Ces différents potentiels n'appartenant pas au domaine de linéarité de la courbe d'étalonnage, ce qui permet de conclure que les ions iodures sont indétectables, car ils sont en très faible concentration, voire inexistants en solution.

Ions iodures par voltamétrie cyclique associé à la technique des ajouts dosés

Compte tenu des faibles teneurs en ions obtenus précédemment dans les eaux de pluie, cinq échantillons de cinq localités ont été sélectionnés sur les treize (13) localités d'études pour la suite des analyses dans le but de confirmer les données de la méthode potentiométrique, à savoir : Boromo, Manga Somgande, Yako et Zorgho. La limite de détection en voltamétrie cyclique étant de l'ordre de $10^{-6} \mathrm{M}$, aucun pic (Figures 7, 8, 9, 10 , et 11) n'est observé sur les différents voltamogrammes des échantillons d'eau de pluie. L'utilisation de la méthode voltamétrique cyclique associée à la technique des ajouts dosés confirme l'absence des ions iodures dans les eaux de pluie, car aucun pic correspondant à ces ions n'est observé sur les différents voltamogrammes des différents échantillons d'eau collectés.

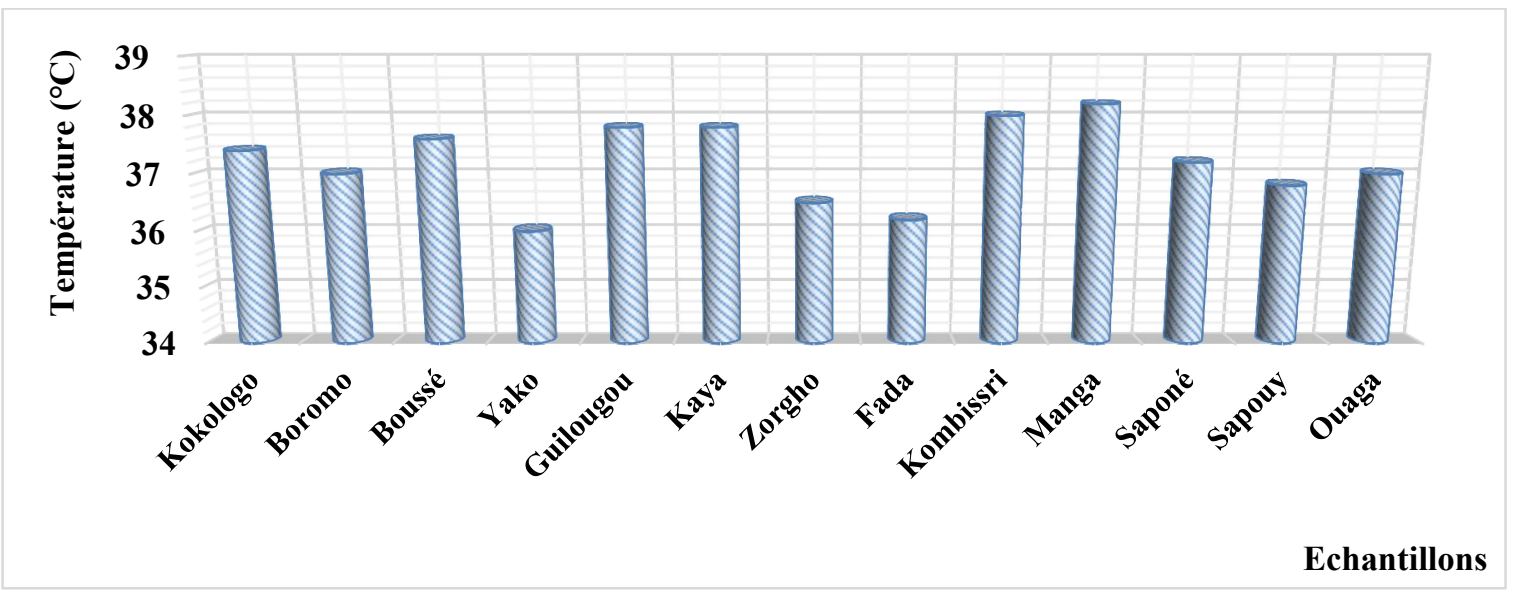

Figure 3 : Variations de la température des échantillons d'eau de pluies provoquées collectés dans les différents sites. 


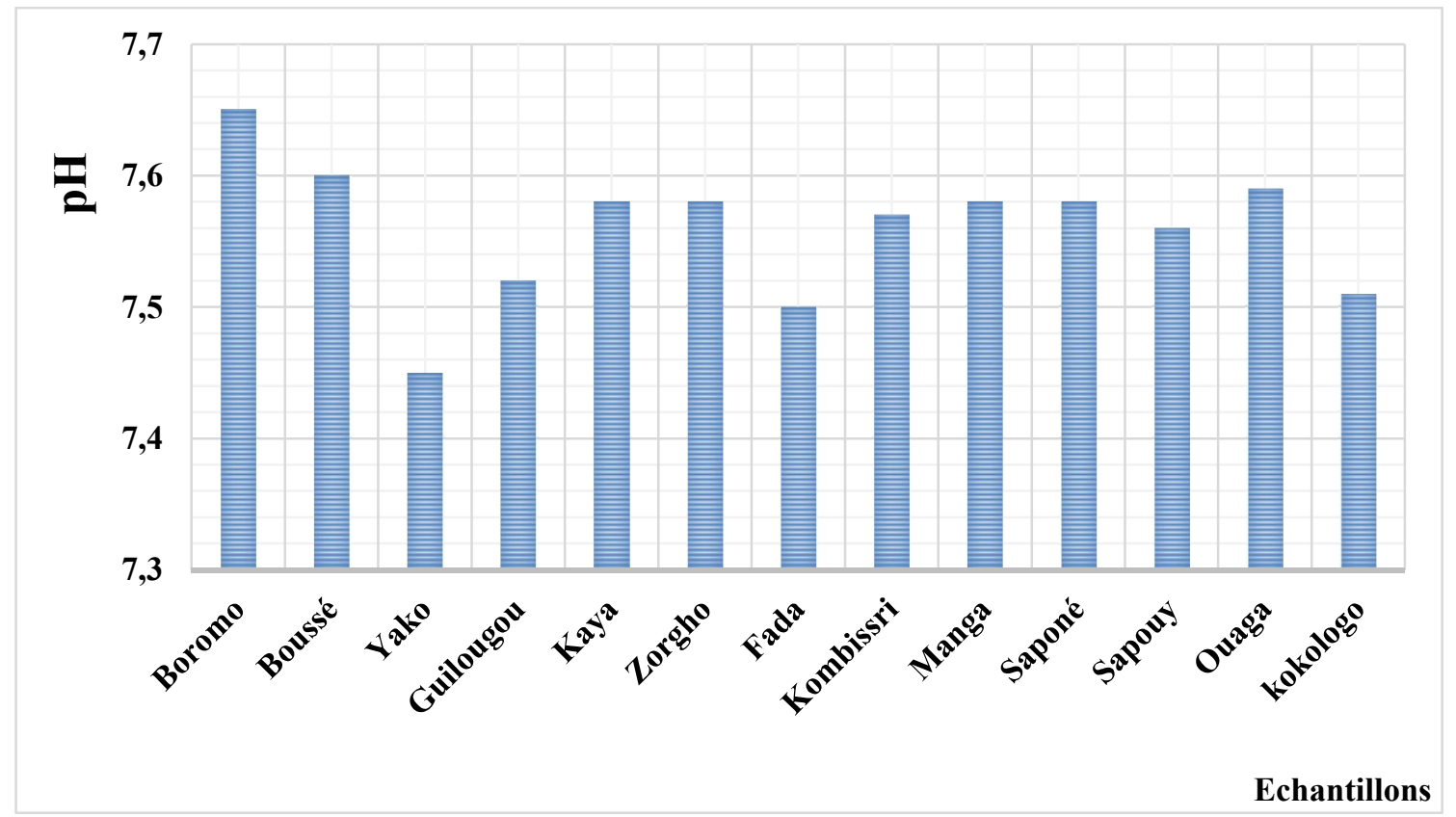

Figure 4 : Variations du potentiel hydrogène des échantillons d'eau de pluies provoquées collectés dans les différents sites.

Tableau 1 : Potentiel E en fonction de la concentration en ions $\mathrm{Ca}^{2+}$ et respectivement $\mathrm{I}^{-}$dans l'électrolyte support $(\mathrm{KCl} 0,1 \mathrm{M})$ respectivement dans l'électrolyte support $\left(\mathrm{KNO}_{3} 0,1 \mathrm{M}\right)$.

\begin{tabular}{lcccc}
\hline$\left[\mathbf{C a}^{2+}\right]$ et $[\mathbf{I}-\mathbf{~} \mathbf{( M )}$ & 0,00001 & 0,0001 & 0,001 & 0,01 \\
\hline $\mathrm{E}(\mathrm{mV}) / \mathrm{REF} 251$ & 39 & 63 & 92 & 121 \\
$\mathrm{E}(\mathrm{mV}) / \mathrm{ECS}$ & -24 & -84 & -144 & -204 \\
\hline $\mathrm{M}:$ molarité. & & &
\end{tabular}

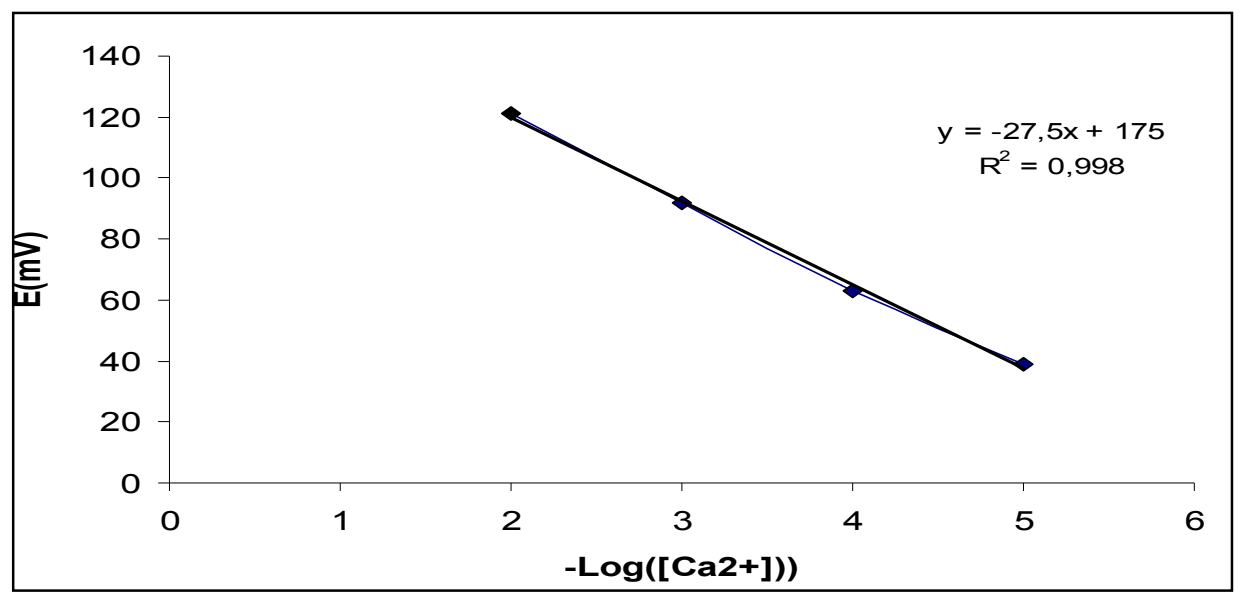

Figure 5 : Courbe d'étalonnage $\mathrm{E}=\mathrm{f}\left(-\log \left[\mathrm{Ca}^{2+}\right]\right)$ de l'électrode spécifique aux ions calcium. 
M.A.MAMAN et al. / Int. J. Biol. Chem. Sci. 11(5): 2521-2536, 2017

Tableau 2 : Analyse de variance des paramètres physicochimiques des eaux de pluie provoquées au Burkina Faso en fonction des sites d'échantillonnage.

\begin{tabular}{|c|c|c|c|c|c|c|c|c|c|c|c|c|c|c|c|}
\hline Sites & Boromo & Boussé & Fada & Guiloungou & Kaya & Kokologo & Kombissiri & Manga & Saponé & Sapouy & $\begin{array}{c}\text { Somgandé } \\
\text { (Ouagadougou) }\end{array}$ & Yako & Zorgho & $\mathbf{P}$ & $\mathbf{F}$ \\
\hline $\mathrm{E}(\mathrm{mV})$ & $47,223^{b c}$ & $38,858^{\mathrm{c}}$ & $48,268^{\mathrm{bc}}$ & $50,755^{\mathrm{abc}}$ & $62,175^{\mathrm{ab}}$ & $56,120^{\mathrm{ab}}$ & $53,542^{\mathrm{abc}}$ & $47,100^{\mathrm{bc}}$ & $47,593^{\mathrm{bc}}$ & $56,160^{\mathrm{ab}}$ & $54343^{\mathrm{abc}}$ & $57,813^{\mathrm{ab}}$ & $66,588^{\mathrm{a}}$ & 0,1004 & ns \\
\hline$\left[\mathrm{Ca}^{2+}\right](\mathrm{mg} / \mathrm{L})$ & $2,451^{\mathrm{a}}$ & $1,415^{\mathrm{a}}$ & $2,703^{\mathrm{a}}$ & $2,635^{\mathrm{a}}$ & $6,55^{\mathrm{a}}$ & $4,183^{\mathrm{a}}$ & $3,605^{\mathrm{a}}$ & $3,453^{\mathrm{a}}$ & $2,370^{\mathrm{a}}$ & $3,928^{\mathrm{a}}$ & $4,810^{\mathrm{a}}$ & $3,263^{\mathrm{a}}$ & $3,948^{\mathrm{a}}$ & 0,379 & ns \\
\hline$\left[\mathrm{I}^{-}\right](\mathrm{mg} / \mathrm{L})$ & $\mathrm{Nd}$ & nd & nd & nd & nd & nd & $\mathrm{Nd}$ & nd & nd & nd & nd & nd & nd & I & l \\
\hline$\left[\mathrm{Ag}^{+}\right](\mathrm{mg} / \mathrm{L})$ & nd & nd & nd & nd & nd & nd & $\mathrm{Nd}$ & nd & nd & nd & nd & nd & nd & / & l \\
\hline $\mathrm{pH}$ & $7,623^{\mathrm{a}}$ & $7,598^{\mathrm{a}}$ & $7,500^{\mathrm{a}}$ & $7,513^{\mathrm{a}}$ & $7,595^{\mathrm{a}}$ & $7,130^{\mathrm{b}}$ & $7,530^{\mathrm{a}}$ & $7,578^{\mathrm{a}}$ & $7,580^{\mathrm{a}}$ & $7,563^{\mathrm{a}}$ & $7,598^{\mathrm{a}}$ & $7,465^{\mathrm{a}}$ & $7,603^{\mathrm{a}}$ & 0,091 & ns \\
\hline $\begin{array}{l}\text { Température } \\
\left({ }^{\circ} \mathrm{C}\right)\end{array}$ & $37^{\mathrm{ef}}$ & $37,6^{\mathrm{bcd}}$ & $36,2^{\mathrm{hi}}$ & $37,8^{\mathrm{abc}}$ & $37,8^{\mathrm{abc}}$ & $37,4^{\text {cde }}$ & $38^{\mathrm{ab}}$ & $38,2^{\mathrm{a}}$ & $37,2^{\operatorname{def}}$ & $36,8^{\mathrm{fg}}$ & $36,9^{\text {ef }}$ & $36^{\mathrm{i}}$ & $36,5^{\mathrm{gh}}$ & 0,028 & $*$ \\
\hline
\end{tabular}

Les résultats sont les moyennes de 3 répétitions $; \mathbf{n d}=$ non déterminé $; \mathbf{P}=$ probabilité $; \mathbf{d d l}=$ degré de liberté $; \mathbf{n s}=$ non significatif au seuil de $5 \% ; *=$ significatif au seuil de $1 \% ; \mathbf{m V}=$ millivolt $; \mathrm{mg} / \mathrm{L}:$ milligramme par litre $; \mathbf{p H}=$ potentiel à hydrogène $; \mathbf{m g} / \mathbf{L}=$ milligramme par litre. Les valeurs qui ont une lettre en commun dans une ligne ne sont pas significativement différentes selon le test de NewmanKeuls au seuil de $5 \%$.

Tableau 3 : Paramètres chimiques des eaux de pluie provoquées au Burkina Faso en fonction des sites et de la période d'échantillonnage.

\begin{tabular}{|c|c|c|c|c|c|c|c|c|c|c|c|c|c|}
\hline \multirow[t]{2}{*}{ Sources de variation } & \multirow[t]{2}{*}{ ddl } & \multicolumn{3}{|c|}{$\mathbf{E}(\mathbf{m V})$} & \multicolumn{3}{|c|}{ pH } & \multicolumn{3}{|c|}{$\left[\mathrm{Ca}^{2+}\right](\mathrm{mg} / \mathrm{L})$} & \multicolumn{3}{|c|}{ Température $\left({ }^{\circ} \mathbf{C}\right)$} \\
\hline & & CM & $\mathbf{P}$ & $\mathbf{F}$ & $\mathbf{C M}$ & $\mathbf{P}$ & $\mathbf{F}$ & $\mathbf{C M}$ & $\mathbf{P}$ & $\mathbf{F}$ & CM & $\mathbf{P}$ & $\mathbf{F}$ \\
\hline Période & 2 & 33,046 & 0,235 & ns & 0,002 & 0,460 & ns & 3,857 & 0,444 & $\mathrm{~ns}$ & 0,238 & 0,075 & ns \\
\hline Site & 12 & 212,433 & 0,100 & ns & 0,006 & 0,091 & ns & 6,541 & 0,379 & $\mathrm{~ns}$ & 1,934 & 0,028 & $*$ \\
\hline Site $\times$ Période & 36 & 29,233 & 0,270 & ns & 0,001 & 0,679 & ns & 1,535 & 0,698 & ns & 0,118 & 0,115 & ns \\
\hline
\end{tabular}

$\mathbf{C M}=$ Carrés moyens $; \mathbf{n s}=$ non significatif ; * significatif au seuil de $5 \% ; \mathbf{p}=$ probabilité $; \mathbf{d d l}=$ degré de liberté. 


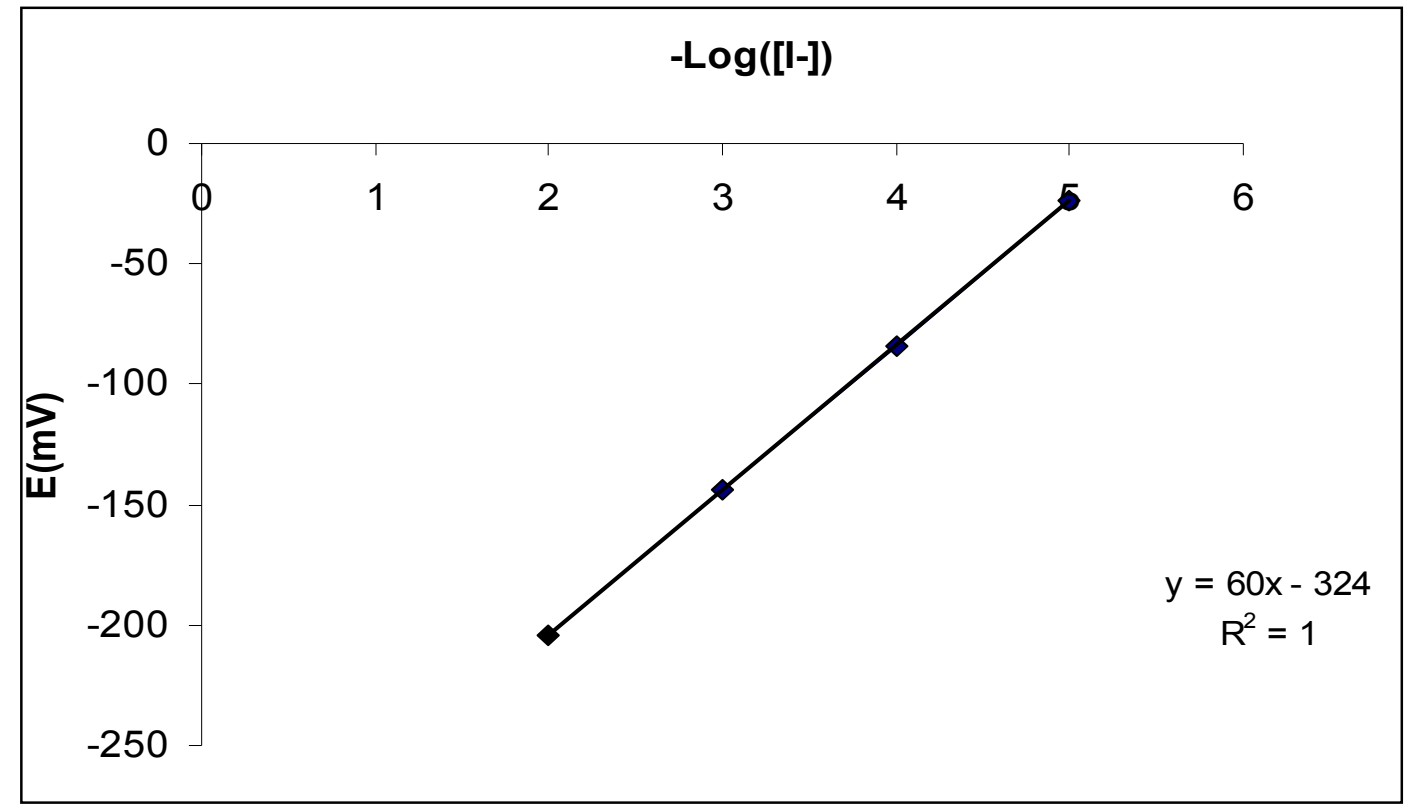

Figure 6: Courbe d'étalonnage $E=f\left(-\log \left[I^{-}\right]\right)$de l'électrode spécifique aux ions iodures.

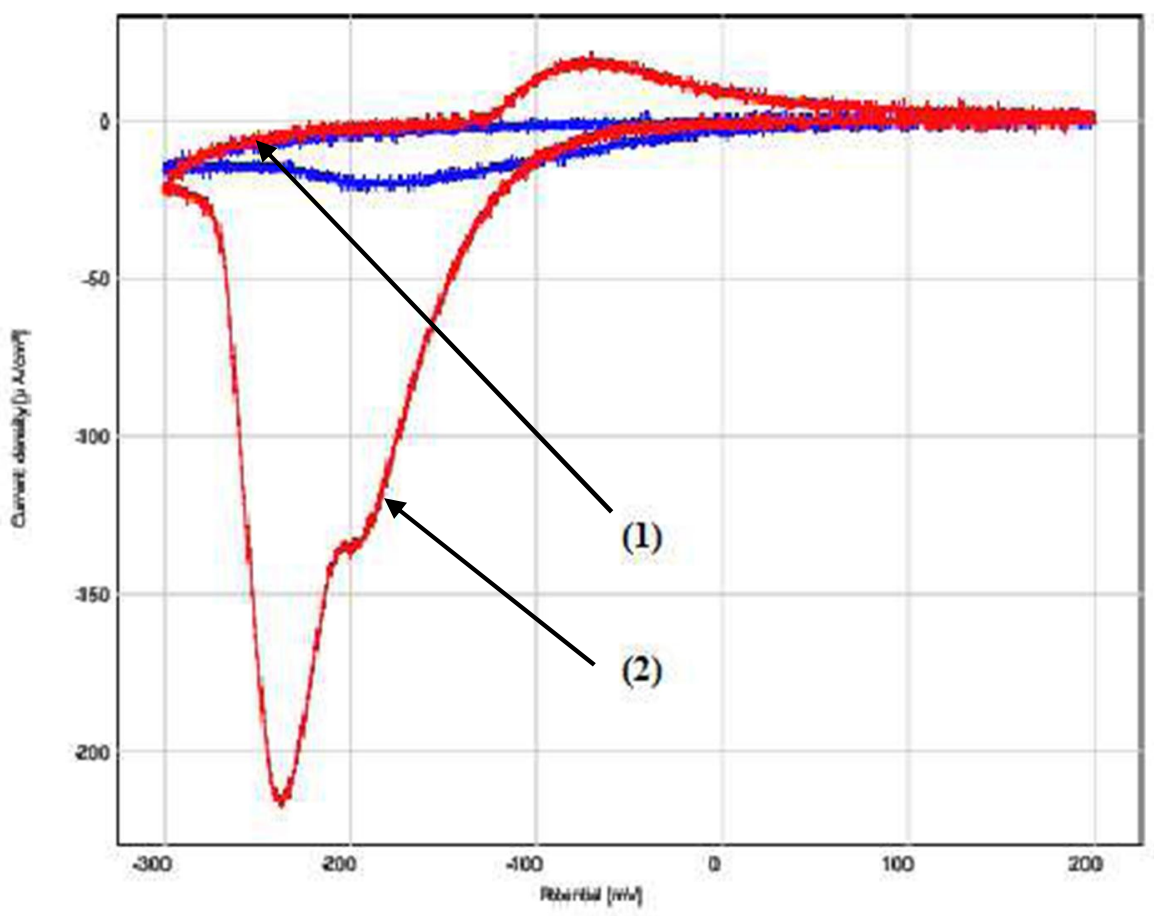

Figure 7 : Dosage des ions iodures des échantillons d'eau de pluie de Zorgho. (1) voltamogramme de base (échantillon d'eau) ; (2) voltamogramme obtenue après ajout de $100 \mu \mathrm{L}$ de $\mathrm{KI} 10^{-3} \mathrm{M}$. 


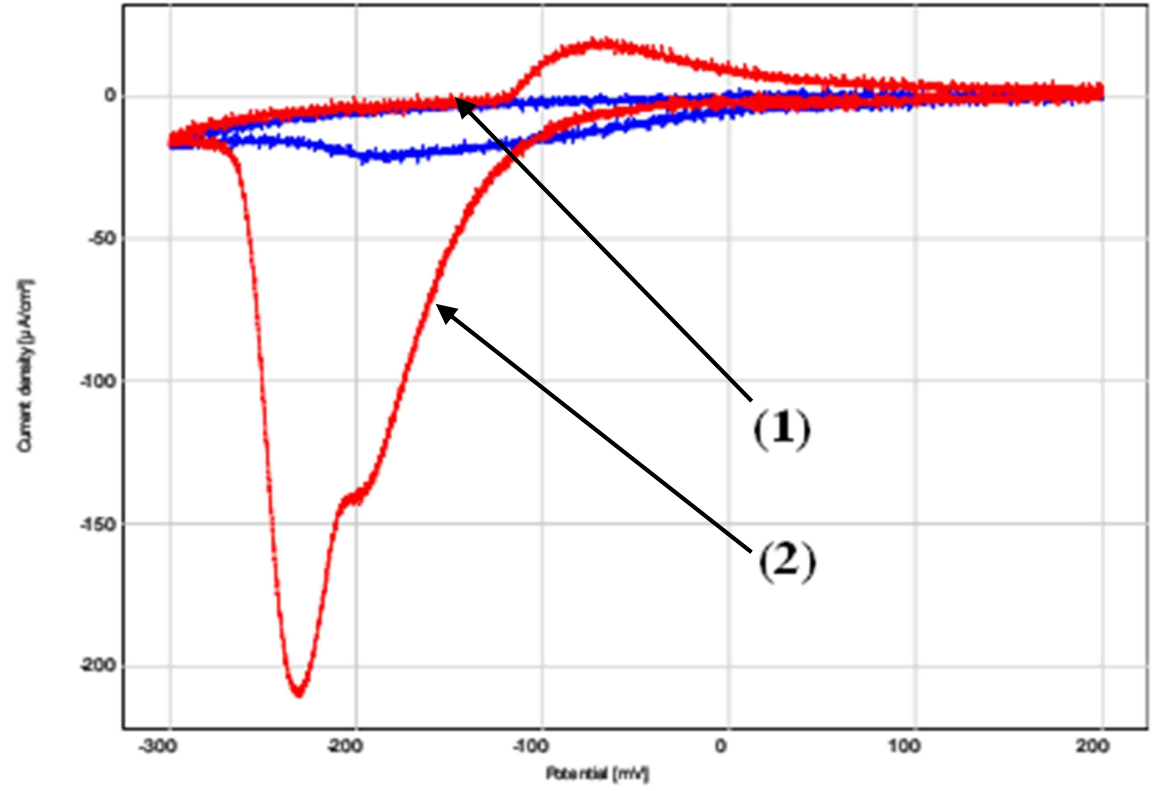

Figure 8 : Dosage des ions iodures des échantillons d'eau de pluie de Yako. (1) voltamogramme de base (échantillon d'eau) ; (2) voltamogramme obtenue après ajout de $100 \mu \mathrm{L}$ de KI $10^{-3} \mathrm{M}$.

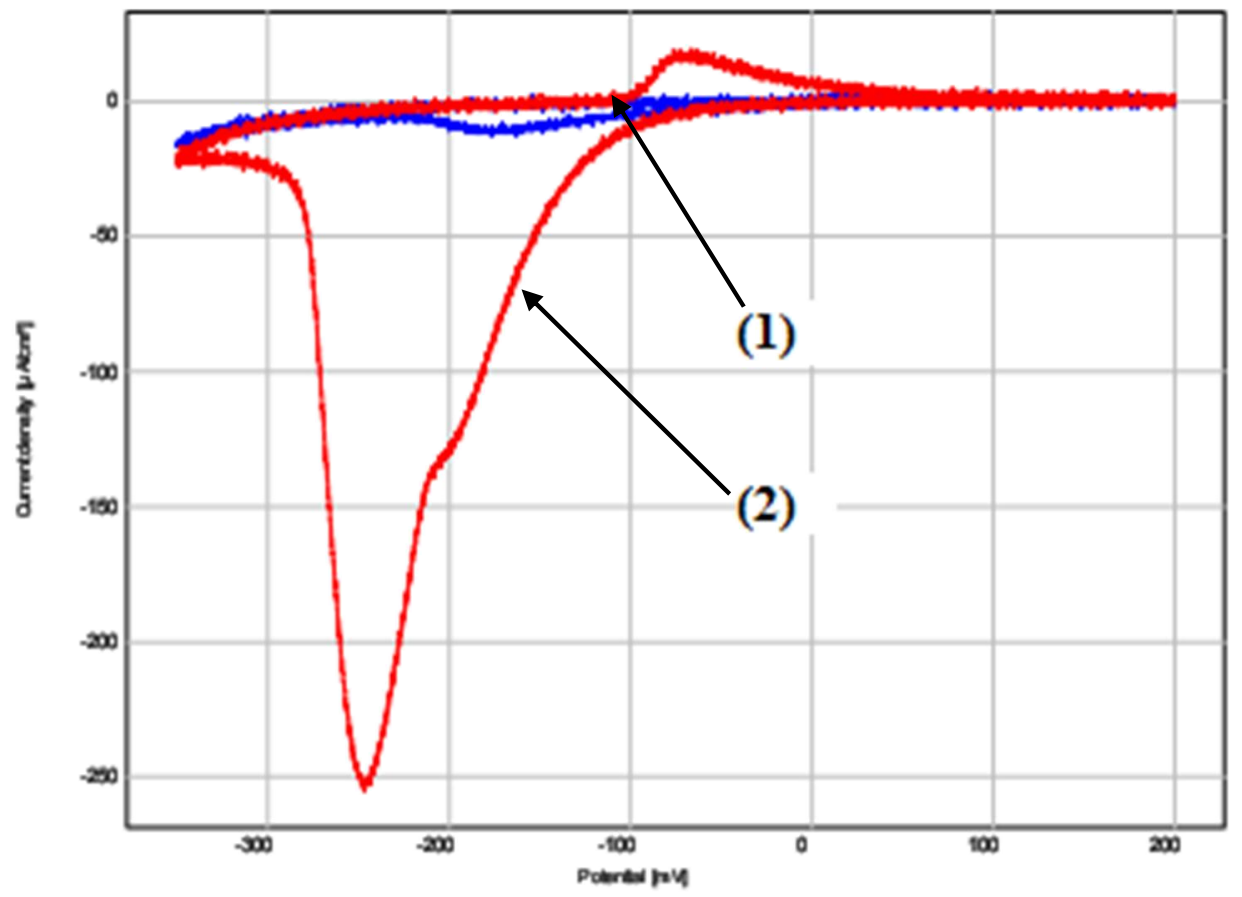

Figure 9 : Dosage des ions iodures des échantillons d'eau de pluie de Somgandé. (1) voltamogramme de base (échantillon d'eau) ; (2) voltamogramme obtenue après ajout de $100 \mu \mathrm{L}$ de KI $10^{-3} \mathrm{M}$. 


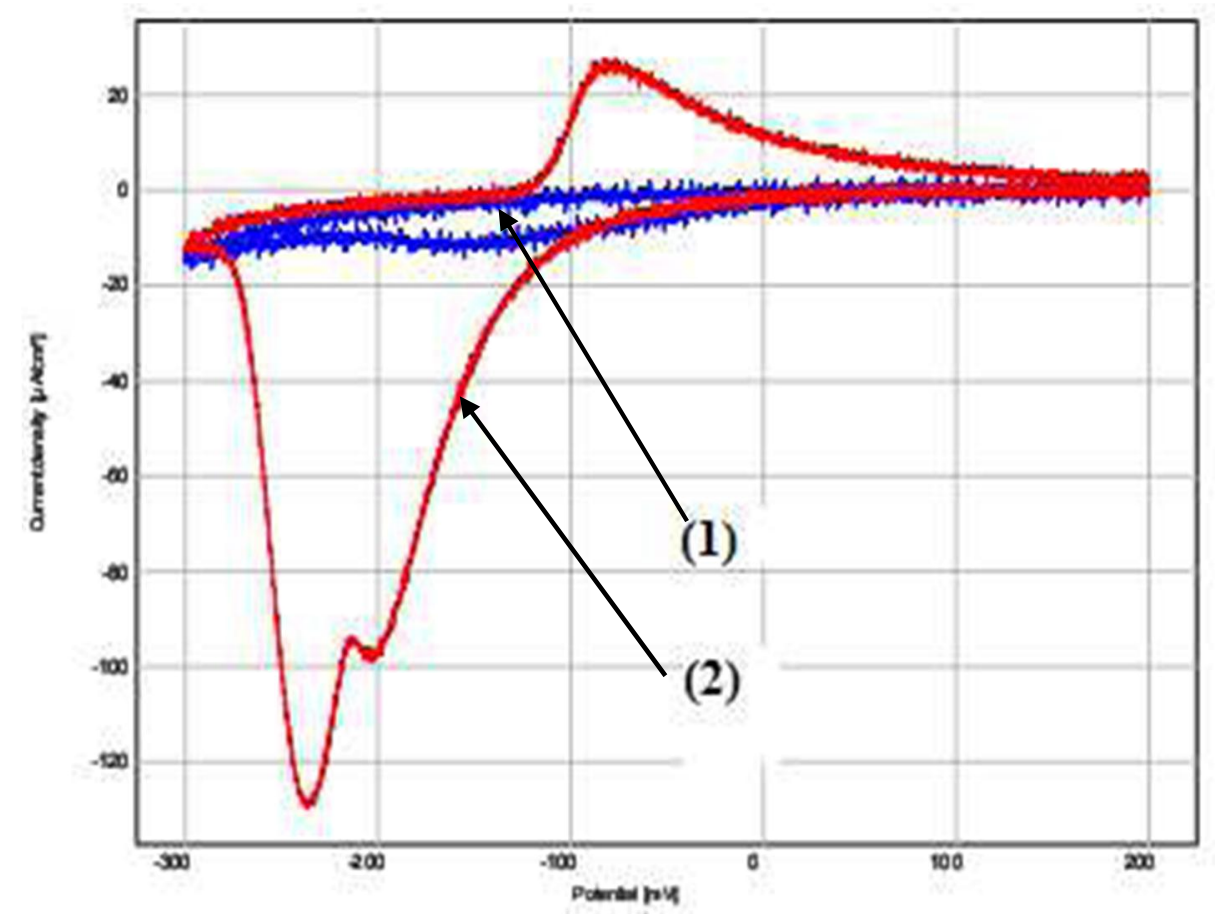

Figure 10 : Dosage des ions iodures des échantillons d'eau de pluie de Manga. (1) voltamogramme de base (échantillon d'eau) ; (2) voltamogramme obtenue après ajout de $100 \mu \mathrm{L}$ de $\mathrm{KI} 10^{-3} \mathrm{M}$.

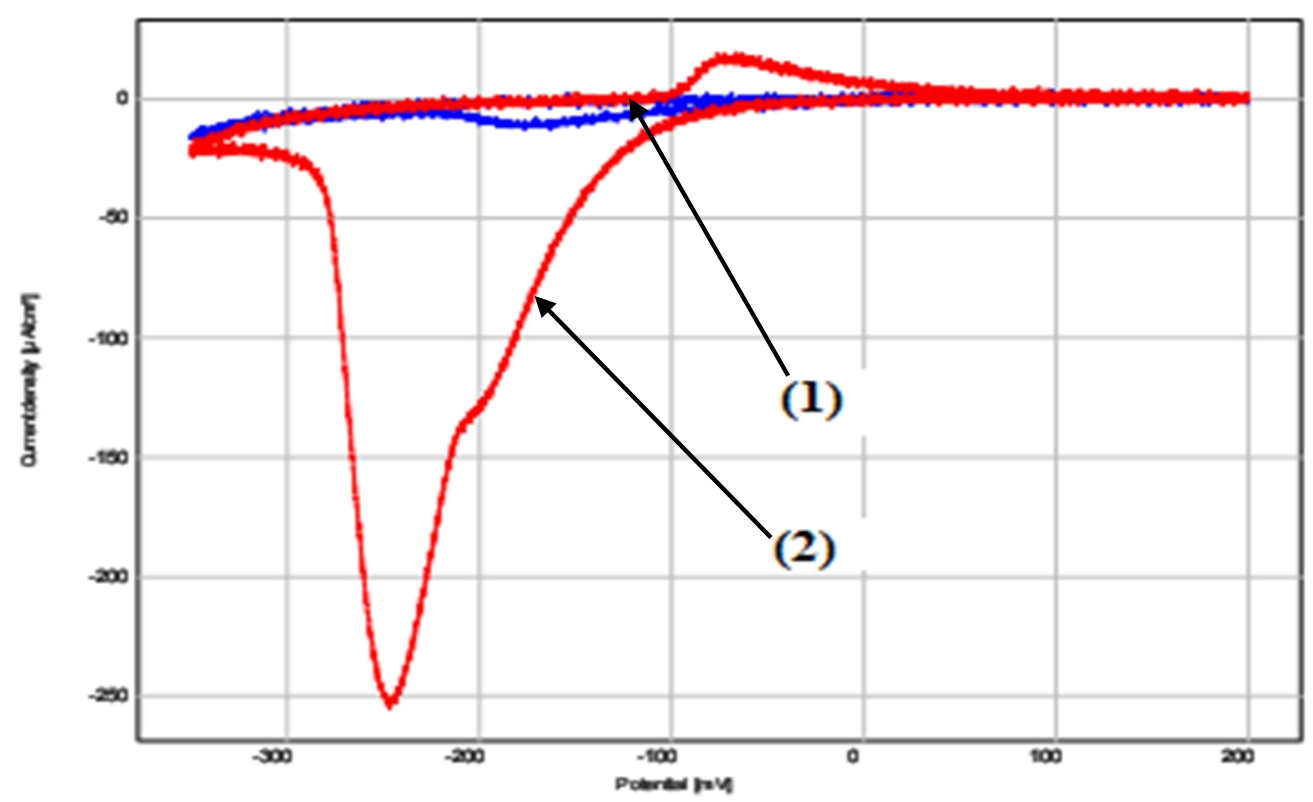

Figure 11 : Dosage des ions iodures des echantillons d'eau de pluie de Boromo. (1) voltamogramme de base (échantillon d'eau) ; (2) voltamogramme obtenue après ajout de $100 \mu \mathrm{L}$ de $\mathrm{KI} 10^{-3} \mathrm{M}$. 
DISCUSSION

Qualité des eaux de pluie

Les résultats analytiques présentés

dans cette étude représentent les déterminations physico-chimiques et minérales analysées en référence à différentes normes en vigueur. Les prélèvements effectués dans le cadre des différentes campagnes pluvieuses de mesure sont de type instantané; ils ne sont ainsi que le reflet de la qualité de l'eau à un moment donné.

\section{Analyses électrochimiques}

$\mathrm{Au}$ plan électrochimique les résultats de cette étude indiquent que les valeurs observées pour les différents paramètres mesurés sont en lien avec la période d'échantillonnage. Les valeurs de températures relevées sont en accord avec la littérature : les eaux souterraines sont moins sensibles aux variations de température que les eaux superficielles (Abdoulkadri et al., 2015). Les valeurs de $\mathrm{pH}$ sont conformes aussi à la norme OMS avec une tendance basique probablement due au manque d'activités industrielles dans les différentes zones (villes) d'étude, mais aussi des premières pluies très chargées de poussières et assez fortement minéralisée (Magnin, 1984). Selon Rodier (2009), ces eaux ne sont pas à priori interdites pour la consommation humaine. Des résultats d'analyse, il ressort clairement que sur un plan d'ensemble, les eaux de pluie contiennent des ions calcium qui sont en dessous de la norme européenne, de même que les ions argent et iodure sont à l'état de trace. Par conséquent, les échantillons d'eau de pluie ne contenant pas d'ions iodures ou s'ils existent, alors ils seraient à l'état de trace donc non décelable par les méthodes utilisées. Les concentrations d'ions iodures admissibles dans l'eau potable varient entre $0,01 \mathrm{mg} / 1$ et $0,14 \mathrm{mg} / 1$ (Somé et al., 2008; Nitiema et al., 2013), d'où l'existence de ces ions à l'état de trace ne constituerait aucun danger. Les résultats du dosage obtenus pour les ions iodures $\left(\mathrm{I}^{-}\right)$sont aussi valables pour les ions argent $\left(\mathrm{Ag}^{+}\right)$. En effet, les ions $\mathrm{Ag}^{+}$et $\mathrm{I}^{-}$provenant de la dissociation de l'iodure d'argent (AgI) libère exactement les mêmes nombres de moles. La concentration maximale admise pour les ions argent est de $10 \mu \mathrm{g} / \mathrm{l}$ selon les normes européennes (Organisation Mondiale de la Santé/UNICEF, 2007).

\section{Conclusion}

Les résultats obtenus dans cette étude indiquent que les eaux de pluie provoquées par l'opération SAAGA dans les 13 localités du Burkina Faso sont relativement de bonne qualité au plan physico-chimique essentiellement en période pluvieuse. En effet, dans ce travail qui est une contribution à l'analyse électrochimique des eaux de pluie provoquées au Burkina Faso, une détermination de la concentration en ions calcium, iodure et argent dans les eaux de pluie provoquées dans différentes localités du Burkina Faso a été effectuée. Les résultats d'analyses électrochimiques (la potentiométrie à intensité nulle et la voltamétrie cyclique associée à la technique des ajouts dosés) ont révélé que les échantillons d'eau de pluie provoquée par le programme dénommé "SAAGA', ne contiennent ni d'ions iodures $\left(\mathrm{I}^{-}\right)$, ni d'ions argent $\left(\mathrm{Ag}^{+}\right)$: les concentrations mesurées de ces ions sont inférieures à $10^{-6} \mathrm{M}$ donc elles se trouvent à l'état de traces après une analyse chimique. Mais, tous les échantillons contiennent de faibles quantités d'ions calcium $\left(\mathrm{Ca}^{2+}\right)$ provenant certainement du calcaire de l'atmosphère. Les opérations d'ensemencement des nuages du programme SAAGA ne seraient ainsi une source de contamination des eaux de pluie et de l'environnement au Burkina Faso, par conséquent pas d'impacts sur l'être humain. Donc elles peuvent être bel et bien consommables par la population locale, les plantes et les animaux.

\section{CONFLITS D'INTÉRÊTS}

Tous les auteurs déclarent qu'il n'y a aucun conflit d'intérêts pour cet article.

\section{CONTRIBUTIONS DES AUTEURS}

MAM a contribué aux différentes collectes des échantillons sur les différents sites d'études, à l'analyse des échantillons et à la rédaction du manuscrit. JS contribué à la conception de la méthodologie de travail et à 
la conception de la carte. DB a contribué dans la correction du projet d'article. MB a été utile au laboratoire, a participé à l'écriture et à l'amélioration du manuscrit. Il a également contribué à la vérification du langage du document. Enfin, JBL a amélioré la section discussion. Il a également fourni les aides financières pendant la collecte (travaux sur le terrain). Tous les auteurs se sont ont contribué sur le choix des sites de prélèvement, la période de collectes des échantillons, les méthodes des analyses électrochimiques et de la méthodologie d'analyse.

\section{RÉFÉRENCES}

Abdoulkadri AM, Boubié G. 2015. Caractérisations physico-chimiques des eaux souterraines de la localité de Yamtenga (Burkina Faso). International Journal of Biological and Chemical Sciences, 9(1): 517 - 533, DOI: http://dx.doi.org/10.4314/ijbcs.v9i1.44.

Ahoussi E, Soro N, Kouassi A, Soro G, Koffi Y, Zade S. 2010. Application des méthodes d'analyses statistiques multivariées à l'étude de l'origine des métaux lourds $(\mathrm{Cu} 2+, \mathrm{Mn} 2+, \mathrm{Zn} 2+$ et $\mathrm{Pb} 2+$ ) dans les eaux des nappes phréatiques de la ville d'Abidjan. International Journal of Biological and Chemical Sciences, 4(5): 1753 - 1765.

Altaher H, Alghamdi A. 2011. Enhancement of Quality of Secondary Industrial Wastewater Effluent by Coagulation Process: A Case Study. Journal of Environmental Protection, 2(9): 1250 1256, DOI: 10.4236/jep.2011.29144.

Beniston M. 2011. Le stockage de l'eau en hivers est de plus en plus important. http://www.letemps.ch/Page/Uuid/d7adc 8b2-33cb-11e0-a2b9-e86504b2dedb: 'Accessed'

Breton A, Vialle C, Montrejaud-Vignoles M, Cecutti C, Vignoles C, Sablayrolles C. 2010. Contribution of car, truck, bus and subway wash station discharges to stormwater pollution (Toulouse, France). Fresenius Environmental Bulletin. 19(9a): 1954-1962, doi: URL : http://www.psp-parlar.de.
Cheng J, Jandik P, Avdalovic N. 2005. Pulsed amperometric detection of sulfide, cyanide, iodide, thiosulfate, bromide and thiocyanate with microfabricated disposable silver working electrodes in ion chromatography. Analytica Chimica Acta. $\quad$ 536(1): 267-274, DOI: http://dx.doi.org/10.1016/j.aca.2004.12.0 39

Chippaux J-P, Houssier S, Gross P, Bouvier C, Brissaud F. 2002. Etude de la pollution de l'eau souterraine de la ville de Niamey, Niger. Bull Soc Pathol Exot., 94(2): 119-123.

De Gouvello B. 2011. L'utilisation de l'eau de pluie à l'intérieur des bâtiments. Les enjeux d'une pratique appelée à se développer. Annales des MinesResponsabilité et Environnement, 3(63): 96 - 101, DOI: 10.3917/re.063.0096.

Dianou D, Poda J N, Sorgho H, Wango S P, Sondo K B, 2003, Hydraulic planning and schistosomiasis: Case of Sourou in Burkina Faso. Journal of Applied Research in Veterinary Medicine, 1(2): 105 - 111 , DOI: http://www.jarvm.com/articles/Vol1Iss2/ Dianou.htm

Dianou D, Savadogo B, Zongo D, Zougouri T, Poda J N, Bado H, Rosillon F. 2011. Qualité des eaux de surface dans la vallée du Sourou : cas des rivières Mouhoun, Sourou, Debe et Gana au Burkina Faso. International Journal of Biological and Chimical Sciences. 5(4): $1571 \quad$ - $\quad 1584, \quad$ DOI: http://dx.doi.org/10.4314/ijbcs.v5i4.22

Fourcade F, Tzedakis T. 2000. Study of the mechanism of the electrochemical deposition of silver from an aqueous silver iodide suspension. Journal of Electroanalytical chemistry. 493(1): 20 27,DOI: http://dx.doi.org/10.1016/ S0022-0728(00)00318-1.

Houelome TMA, Adandedjan D, Chikou A, Imorou I T, Bonou C, Youssao I, Laleye P. 2016. Evaluation de la qualité des eaux des ruisseaux du cours moyen de la rivière Alibori par l'étude des macroinvertébrés benthiques dans le bassin cotonnier du Benin (Afrique de 
l'Ouest). International Journal of Biological and Chemical Sciences. 10(6): $2461 \quad$ - 2476, DOI: http://dx.doi.org/10.4314/ijbcs.v10i6.5.

Journal Officiel de la République Française J. 2008. Arrêté du 21 août 2008 relatif à la récupération des eaux de pluie et à leur usage à l'intérieur et à l'extérieur des bâtiments, NOR: DEVO0773410A, Version consolidée au 19 avril 2017. JORF. 0201(5): 13585.

Kabore A, Savadago B, Rosillon F, Straore A S, Dianou D. 2013a. Optimisation de l'efficacité des graines de moringa oleifera dans le traitement des eaux de consommation en Afrique subsaharienne: Cas des eaux du Burkina Faso. Revue des sciences de l'eau/Journal of Water Science, 26(3): 209-220.

Kabore A, Savadogo B, Otoidobiga H C, Rosillon F, Traore A S, Dianou D, 2013b, Efficacité des graines de MORINGA OLEIFERA dans le traitement des eaux de consommation en Afrique sub saherienne : cas des eaux du Burkina Faso. Annales de l'Université de Ouagadougou - Série C. 009108 - 130.

Kabore A, Savadogo B, Rosillon F, Traore A

S, Dianou D. 2013. Effectiveness of Moringa oleifera Defatted Cake versus Seed in the Treatment of Unsafe Drinking Water: Case Study of Surface and Well Waters in Burkina Faso. Journal of Water Resource and Protection. 5(11): 1076 - 1086, DOI: http://dx.doi.org/10.4236/jwarp.2013.511 113.

Kissinger PT, Heinemann WR. 1984. Laboratory Techniques in Electroanalytical Chemistry. Marcel Dekker: New York.

Kouam KGR, Mpakam HG, Ndonwy SA, Bopda SLD, Ekodeck GE. 2006. Gestion intégrée des ressources en eau et objectifs du millénaire pour le développement en Afrique : Cas du Cameroun. Vertigo, 7(2): 1-9, DOI: 10.4000/vertigo.2319

Koukounari A, Gabrielli AF, Toure S, Elisa B-O, Zhang Y, Sellin B, Donnelly CA,
Alan F, Webster JP. 2007. Schistosoma haematobium Infection and Morbidity Before and After Large-Scale Administration of Praziquantel in Burkina Faso. Journal of Infectious Diseases, 196(5): 659 -669, DOI: 10.1086/520515.

Lautenschlager K, Boon N, Wang Y, Egli T, Hammes F. 2010. Overnight stagnation of drinking water in household taps induces microbial growth and changes in community composition. Water Research, 17(44): 4868 - 4877, DOI: 10.1016/j.watres.2010.07.032.

Magnin F. 1984. Quelques observations sur le $\mathrm{pH}$ des eaux de pluie en Provence. Méthodes et premiers résultats. Méditerranée, troisième série, 52(3): 79 - 84, DOI: 10.3406/medit.1984.2259

Maman MA. 2008. Analyses des eaux par potentiométrie et voltamétrie cyclique associée à la technique des ajouts et dosées : cas des eaux de pluies du “ PROGRAMME SAAGA”. Mémoire de DEA de l'Université de Ouagadougou. $54 \mathrm{p}$.

Mathieu P, Monnet C, 1971, Physico-chimie des eaux de pluie en savane et sous fort en milieu tropical. Cah. Orstom, sér. Géol., III: 9-30.

Nitiema LW, Savadogo B, Zongo D, Kabore A, Poda JN. 2013. Microbial Quality of Wastewater Used in Urban Truck Farming and Health Risks Issues in Developing Countries: Case Study of Ouagadougou in Burkina Faso. Journal of Environmental Protection, 4(6): 575 584 ,

DOI: http://dx.doi.org/10.4236/jep.2013.46067

Organisation Mondiale de la Santé. 2000. Directive de Qualité pour l"Eau de Boisson, Critères d"Hygiène et Documentation à l"Appui (vol 2, $2^{\mathrm{e}}$ édn). Organisation Mondiale de la santé; 1050 p.

Organisation Mondiale de la Santé/UNICEF O. 2007. Atteindre l'OMD relatif à l'eau potable et à l'assainissement le défi urbain et rural de la décennie. UNICEF, $41 \mathrm{p}$. 
Rodier J, Legube B, Merlet N. 2009. L'Analyse de l'Eau (9 $9^{\mathrm{ème}}$ édn). Ed. Dunod : Paris.

Rosillon F, Savadogo B, Kabore A, BadoSama H, Dianou D, 2012, Attempts to Answer on the Origin of the High Nitrates Concentrations in Groundwaters of the Sourou Valley in Burkina Faso. Journal of Water Resource and Protection, 4(08): 663, DOI: http://dx.doi.org/10.4236/jwarp.2012.480 77.

Rosillon F, Savadogo B, Kabore A, Badosama H, Dianou D, 2012, Estimation of the nitrates contents in waters by using reagent strips: An environment education exercise in the Sourou Valley in Burkina Faso. VertigO, 12(2): 1 - 13, DOI: 10.4000/vertigo.12274.

Sablayrolles C, Breton A, Vialle C, Vignoles C, Montréjaud-Vignoles M, 2011, Priority organic pollutants in the urban water cycle (Toulouse, France). Water Science and Technology, 64(3): 541-556, DOI: $10.2166 /$ wst.2011.580.

Sanou AG, Dembele KD, Ouédraogo I, Dakuo D. 2016. Problématique de mise en œuvre du système de riziculture intensif dans les périmètres rizicoles irrigués de karfiguéla et de la vallée du kou au Burkina Faso. International Journal of Biological and Chimical Sciences 10(6): 2693 -2709., DOI http://dx.doi.org/ 10.4314/ijbcs.v10i6.23.

Savadogo B, Kaboré A, Zongo D, Poda JN, Bado H, Rosillon F, Dianou D. 2013. Problematic of drinking water access in rural area: case study of the Sourou Valley in Burkina Faso. Journal of Environmental Protection, 4(1): 31 - 50, DOI: 10.4236/jep.2013.41004.

Sawadogo J, Bougouma M, Legma JB, Delplancke OMP. 2015, Determination of stoichiometry from polycrystalline powders of transition metals lamellar dichalcogenides: MoSe2, WSe2, obtained by synthesis in laboratory. International Journal of Biological and
Chemical Sciences, 9(1): 454 - 471, DOI: http://dx.doi.org/10.4314/ijbcs.v9i1.39.

Shain I, Perone S, 1961, Application of stripping analysis to the determination of iodide with silver microelectrodes. Analytical Chemistry, 33(3): 325, DOI: 10.1021/ac60171a004.

Somé K, Dembélé Y, Somé L, Millogo RJ. 2008. Pollution agricole des eaux dans le bassin du Nakanbé : cas des réservoirs de Loumbila et de Mogtédo au Burkina Faso. Sud Sciences et Technologies. 16(1): 14 - 22.

Sunda Makuba T. 2012. Contribution à la désinfection de l'eau par photosensibilisation avec des extraits de plantes. Thèse de l'Université de Liège, Arlon, Belgique.

Turton A, Solomon H, 2000, Water Wars: Enduring Myth Or Impending Reality? 2. 176.

Vialle C, Sablayrolles C, Lovera M, Huau MC, Montréjaud-Vignoles M. 2011. Modelling of a roof runoff harvesting system: the use of rainwater for toilet flushing. Water Science and Technology: Water Supply., 11(2): 151-158, DOI: 10.2166/ws.2011.031.

Vialle C, Sablayrolles C, Vignoles C, Montréjaud-Vignoles M. 2010. Impact of carwash discharge on stormwater quality (Toulouse, France). Water Science and Technology, 62(12): 27372746, DOI: $10.2166 /$ wst.2010.929.

World Health Organization W. 2004. Guidelines for Drinking Water Quality ( $3^{\text {rd }}$ édn). WHO Library Cataloguing-inPublication Data : Geneva; 1 - 540.

Yao MK, Agbri L, Bamba SB, Soro BM, Trokourey A. 2016. Prédiction de l'évolution spatio-temporelle du fer à l'exutoire d'un fleuve tropical : cas du fleuve Sassandra à Gaoulou (Côte d'ivoire). International Journal of Biological and Chemical Sciences. 10(6): $2770 \quad$ - 2782, DOI: http://dx.doi.org/10.4314/ijbcs.v10i6.29. 